\title{
Dual molecular imaging for targeting metalloproteinase activity and apoptosis in atherosclerosis: molecular imaging facilitates understanding of pathogenesis
}

\author{
Nezam Haider, PhD, ${ }^{a}$ Dagmar Hartung, $M D,{ }^{a}$ Shinichiro Fujimoto, $M D, P h D,{ }^{a}$ \\ Artiom Petrov, PhD, ${ }^{a}$ Frank D. Kolodgie, PhD, ${ }^{b}$ Renu Virmani, MD, ${ }^{b}$ \\ Satoru Ohshima, MD, PhD, ${ }^{a}$ Han Liu, MS, ${ }^{a}$ Jun Zhou, MD, ${ }^{a}$ Ai Fujimoto, MD, ${ }^{a}$ \\ Atsuko Tahara, $M D,{ }^{\text {a }}$ Leo Hofstra, $M D,{ }^{c}$ Navneet Narula, $M D,{ }^{d}$ \\ Chris Reutelingsperger, $\mathrm{PhD},{ }^{\mathrm{e}}$ and Jagat Narula, $\mathrm{MD}, \mathrm{PhD}^{\mathrm{a}}$
}

Background. Macrophage apoptosis and MMP activity contribute to vulnerability of atherosclerotic plaques to rupture. By employing molecular imaging techniques, we investigated if apoptosis and MMP release are interlinked.

Methods. Atherosclerosis was produced in rabbits receiving high-cholesterol diet (HC), who underwent dual radionuclide imaging with ${ }^{99 m}$ Tc-labeled matrix metalloproteinase inhibitor (MPI) and ${ }^{111}$ In-labeled annexin A5 (AA5) using micro-SPECT/CT. \% ID/g MPI and AA5 uptake was measured, followed by histological characterization. Unmanipulated animals were used as disease controls. Correlation between MPI and AA5 uptake was undertaken and relationship confirmed in culture study of activated THP-1 monocytes.

Results. MPI and AA5 uptake was best visualized in HC diet animals $(n=6)$ and reduced significantly after fluvastatin treatment $(n=4)$ or diet withdrawal $(n=3)$. \% ID/g MPI $(.087 \pm .018 \%)$ and AA5 $(.03 \pm .01 \%)$ uptake was higher in HC than control $(\mathrm{n}=6)$ animals $(.014 \pm .004 \%, P<.0001 ; .0007 \pm .0002 \%, P<.0001)$, and reduced substantially after diet or statin intervention. There was a significant correlation between MPI and AA5 uptake $(r=.62$, $P<.0001$ ), both correlated with pathologically verified MMP-9 activity, macrophage content, and TUNEL staining. In vitro studies demonstrated MMP-9 release in culture medium from apoptotic THP-1 monocytes.

Conclusions. The present study suggests that apoptosis and MMP are interrelated in atherosclerotic lesions and the targeting of more than one molecular candidate is feasible by molecular imaging. (J Nucl Cardiol 2009;16:753-62.)

Key Words: Radionuclides $\cdot$ SPECT • vulnerable atherosclerotic plaque

From the Department of Cardiology, ${ }^{a}$ Irvine School of Medicine, University of California, Irvine, CA; Cardiovascular Pathology Institute, ${ }^{\mathrm{b}}$ Gaithersburg, MD; Department of Cardiology, ${ }^{\mathrm{c}}$ University Hospital Maastricht, Academic University of Maastricht, Maastricht, The Netherlands; Department of Pathology, ${ }^{\mathrm{d}}$ Irvine School of Medicine, University of California, Irvine, CA; Department of Biochemistry, ${ }^{\text {e }}$ University Hospital Maastricht, Academic University of Maastricht, Maastricht, The Netherlands.

Nezam Haider, Dagmar Hartung, and Shinichiro Fujimoto contributed equally to this study.

Received for publication Feb 8, 2009; final revision accepted May 23, 2009.

Reprint requests: Artiom Petrov, PhD, Department of Cardiology, Irvine School of Medicine, University of California, C116 Med Sci I, Irvine, CA 92697; adpetrov@uci.edu.

$1071-3581 / \$ 34.00$

Copyright $\odot 2009$ The Authors(s). This article is published with open access at Springerlink.com.

doi:10.1007/s12350-009-9107-8
See related editorial, pp. 686-688

\section{INTRODUCTION}

In two-thirds of patients presenting with acute coronary syndromes, coronary thrombosis is associated with rupture of the fibrous cap of an atherosclerotic plaque. ${ }^{1}$ The triggers for the rupture include prevalence of monocyte-macrophage infiltration and their apoptosis as well as cytokine production including matrix metalloproteinases (MMP). Almost one half of the macrophages at the site of plaque rupture demonstrate evidence of apoptosis with activation of caspase- $1 .{ }^{2}$ In 
vitro experiments have confirmed the role of caspase- 1 in induction of apoptosis and the use of caspase-1 inhibitor (YVAD) substantially reduced the apoptosis of macrophages in an experimental model of atherosclerosis. ${ }^{3}$ On the other hand, macrophage infiltration in the plaque is associated with MMP expression and in turn to degradation of extracellular matrix and cap rupture. ${ }^{4}$

In addition to acting as a trigger to cap rupture, macrophage apoptosis and MMP activity contribute to plaque vulnerability. Apoptosis of macrophages in necrotic core ${ }^{5}$ leads to enlargement of the core size; greater the necrotic core volume more prone is the plaque to rupture. ${ }^{6}$ On the other hand, the increase in MMP in the necrotic core promotes expansive outward remodeling of the plaque, ${ }^{7}$ and renders the plaque susceptible to rupture. These two processes, which are independently critical for plaque rupture, are both related to macrophage infiltration and it is logical to presume that these processes are interrelated. The apoptosis of cellular components in an atherosclerotic plaque is no longer considered an inert process. Fas-induced apoptosis of smooth muscle cell (SMC) has been shown to produce cytokines such as monocyte-chemoattractant protein 1 (MCP-1), CINC/IL-8, and other pro-inflammatory genes resulting in an extensive macrophage infiltration. ${ }^{8}$ Similar to the SMC-related release of cytokines, it is possible that apoptosis of macrophages may also be associated with secretory activity.

To evaluate if apoptosis of macrophages is linked to MMP and to determine the correlation of macrophage apoptosis and MMP release in vivo, we performed noninvasive imaging with ${ }^{111}$ In-annexin A5 (AA5; as a marker of apoptosis) and ${ }^{99 \mathrm{~m}}$ Tc-labeled broad-spectrum matrix metalloproteinase inhibitor (MPI; as a marker of MMP activity) in rabbits with experimentally induced atherosclerotic lesions. We also evaluated the interrelation by inducing apoptosis of activated monocytes and investigating whether the MMP activity was increased in the culture medium.

\section{METHODS}

Noninvasive imaging was performed in atherosclerotic rabbits for the detection of apoptosis process and MMP activity by employing ${ }^{111}$ In-AA5 (which targets phosphatidyl serine expression on the apoptotic cells) and ${ }^{99 \mathrm{~m}}$ Tc-MPI (which binds to activated catalytic domain of MMP). The correlation of the two processes was confirmed in the in vitro experiments using PMA-activated human monocytic leukemic cell line, THP-1. The THP-1 cells were transfected with caspase-1 gene, and MMP production was determined in the culture medium. MMP-9 production and activity were measured by gel zymography as reported previously by ELISA. ${ }^{9}$ The effects of caspase-1 inhibitor and endoplasmatic reticulum (ER) stress inducers were also evaluated.
Atherosclerosis was produced experimentally in rabbits by aortic deendothelialization and high-cholesterol (HC) diet. To obtain a large range of macrophage infiltration, apoptosis, and MMP production in the atherosclerotic lesions, the hypercholesterolemic rabbits were subjected to dietary modification or treated with fluvastatin. This protocol conforms to the Guidelines for the Care and Use of Laboratory Animals by the US National Institute of Health (NIH Publication No. 8523, revised 1996) and has been approved by the Institutional Laboratory Animal Care and Use Committee at University of California, Irvine.

\section{In Vivo Experimental Design}

The in vivo study was performed in 19 New Zealand white (NZW) rabbits. Of the 19 rabbits, 13 rabbits were subjected to balloon deendothelialization of their abdominal aorta and were fed $\mathrm{HC}$ diet for 4 months to induce atherosclerotic lesions. Of these, 3 atherosclerotic rabbits were returned to normal chow in the last fourth month and 4 received fluvastatin ( $1 \mathrm{mg} / \mathrm{kg}$ p.o., once a day) in the last month. Dual imaging was performed after simultaneous intravenous administration of Tc-99m-labeled MPI $(257.2 \pm 3.7 \mathrm{MBq} / 6.95 \pm 0.10 \mathrm{mCi})$ and In-111-labeled annexin A5 $(17.0 \pm 6.29 \mathrm{MBq} / 0.46 \pm 0.17$ $\mathrm{mCi})$. The remaining 6 rabbits were left unmanipulated for 4 months and fed normal chow. These animals were used for dual imaging as disease controls.

Induction of atherosclerosis. Male NZW rabbits (2.5-3.5 kg) obtained from Western Oregon Breeding Laboratories (Philomoth, OR) were started on $0.5 \%$ cholesterol and $6 \%$ peanut oil diet. One week later, balloon deendothelialization of the abdominal aorta was performed with a $4 \mathrm{~F}$ Fogarty embolectomy catheter (12-040-4F; Edwards Lifesciences LLC, Irvine, CA) as described previously. ${ }^{10}$ For this purpose, animals were anesthetized with a mixture of ketamine and xylazine. The right femoral artery was surgically exposed, an embolectomy catheter was introduced through the femoral arteriotomy and advanced retrograde in the aorta up to the level of the diaphragm. The catheter was inflated and endothelial denudation of the abdominal aorta was performed by pulling down the inflated catheter to the bifurcation of the aorta. Three such passes were made. The femoral artery was then ligated, and the incision site closed. The high-cholesterol, high-fat diet was continued for 15 more weeks.

Imaging agents and radiolabeling. Two targeting agents radiolabeled with two different isotopes were employed for imaging. First, MPI (RP-805, kind gift of Lantheus Imaging Inc., North Billerica, MA) radiolabeled with technetium-99m was used. The chemical structure of MPI has been reported previously. ${ }^{9,11}$ MPI binds specifically to the activated catalytic domain of a broad range of MMP and not other proteases, ${ }^{12}$ and therefore has high enzyme inhibitory profile. ${ }^{9}$ For radiolabeling of MPI, the contents of the vehicle vial were dissolved in $0.5 \mathrm{~mL}$ of $0.9 \%$ sterile saline. The clear solution was transferred to a vial containing 25-35 $\mu \mathrm{g}$ of MPI. Subsequently the mixture was incubated for 10 minutes to dissolve all particles and $\sim 3.33 \mathrm{GBq}(90 \mathrm{mCi} / \mathrm{mL})$ of ${ }^{99 \mathrm{~m}} \mathrm{Tc}$ pertechnetate was added. The reaction vial was heated at $100^{\circ} \mathrm{C}$ 
for 10 minutes. Then HPLC analysis was performed to check the radioefficiency, which revealed product peak of $>97 \%$.

Second, AA5 binds to phosphatidylserin expressed on the surface of apoptotic cells with a nanomolar affinity. Normally, AA5 has been radiolabeled with Tc-99m and employed successfully for experimental and clinical imaging of apoptosis noninvasively. For this study, we developed a novel imaging strategy by labeling AA5 with indium-111. For radiolabeling AA5, $55.5 \mathrm{MBq}(1.5 \mathrm{mCi})$ of ${ }^{111} \mathrm{In}$ was added to $30 \mu \mathrm{g}$ of AA5-DTPA conjugate (kindly provided by PharmaTarget Inc, Maastricht, Netherlands). After 30 minutes of incubation, instant thin layer chromatography was performed.

In vivo and ex vivo imaging protocols. Radionuclide imaging was performed immediately and 4 hours after administration of radiotracers using a dual-head microSPECT gamma camera combined with micro-CT (X-SPECT, Gamma Medica, Inc., Northridge, CA). SPECT images of the aorta were acquired in a $64 \times 64$ matrix, 32 stops at 20 seconds (at 0 hour) or 120 seconds (at 4 hours) per stop first at $140 \mathrm{keV}$ photopeak of ${ }^{99 \mathrm{~m}} \mathrm{Tc}$ with $15 \%$ windows using a low-energy, high-resolution parallel-hole collimator and second at $247 \mathrm{keV}$ photopeak of ${ }^{111}$ In with $15 \%$ windows using a medium energy parallel-hole collimator. Immediately after SPECT imaging, a micro-CT scan was acquired using an X-ray tube operating at $50 \mathrm{kVp}$ and $0.6 \mathrm{~mA}$. Images were captured for 2.5 seconds per view for 256 views in $360^{\circ}$ rotation. After transferring to $256 \times 256$ matrix, the micro-SPECT images and micro-CT tomographic studies were fused. Animals were killed after in vivo imaging, with an overdose of sodium pentobarbital $(120 \mathrm{mg} / \mathrm{kg})$. Ex vivo gamma imaging of the excised aortas was performed. The explanted aortas were imaged for 30 minutes. After ex vivo imaging, the aortas were segmented at $1-\mathrm{cm}$ intervals, weighed, and gamma counted in an automatic welltype gamma counter (Perkin Elmer Wallac Inc., Gaithersburg, MD) for calculation of the percent total injected dose per gram tissue $(\% \mathrm{ID} / \mathrm{g})$ uptake. Aortas were then preserved for histologic and immunohistochemical investigation.

Tissue samples of the main organs were used for calculation of the $\% \mathrm{ID} / \mathrm{g}$ uptake to evaluate the biodistribution. To correct for the radioactive decay and permit calculation of the concentration of radioactivity as a fraction of the administered dose, aliquots of the injected dose were counted simultaneously.

Histological and immunohistochemical evaluation. One-half of every $1 \mathrm{~cm}$ aortic segment was snap frozen and the other half was fixed overnight with $4 \%$ paraformaldehyde in PBS, $\mathrm{pH} 7.4$ at $4^{\circ} \mathrm{C}$. The first half of the each specimen was subdivided into three equidistant sections, dehydrated in a graded series of ethanol, and embedded in paraffin for further processing in triplicate. Paraffin blocks were cut in $4 \mu \mathrm{m}$ thick sections, floated on a water bath containing deionized water $\left(43^{\circ} \mathrm{C}\right)$, and mounted on vectabond reagent-treated slides (Vectabond, SP-1800, Vector Labs, Burlingame, CA). After deparaffinization by heating $\left(25\right.$ minutes at $56^{\circ} \mathrm{C}$ ) and dehydration using xylene and graded series of ethanol, the tissue sections were stained with hematoxylin \& eosin and Movat pentachrome stains. Histologic specimens were analyzed using a classification scheme based on the recommendations of the American Heart Association (AHA). ${ }^{13}$

As described previously, ${ }^{9,14}$ immunohistochemistry was performed by standard staining procedures. Smooth muscle cell (SMC) was identified using a primary antibody against $\alpha$-actin isotypes (MAB1420, 1:10,000, R\&D Systems, Minneapolis, MI) and macrophages by RAM-11 (M 0633, 1:3,000, DAKO, Carpinteria, CA). For MMP-9 staining in atherosclerotic lesions monoclonal MMP-9 (gelatinase-B, IM37, 1:15,000, Calbiochem) antibody was used. Color reaction was developed by Novared substrate-chromogen system (SK-4800, red color, Vector Lab) and diaminobenzidine kit (SK-4100, $\mathrm{DAB}$, brown color, Vector Lab, Burlingame, CA). The sections were counterstained with Gill's hematoxylin. Sections incubated in parallel without primary antibody or control IgG were used as negative control. Immunostaining was observed (Zeiss Axiovert-200 microscope, Carl Zeiss, Thornwood, NY) and images captured (Zeiss Axiocam high-resolution digital color camera, $1,300 \times 1,030$ pixels using Axiovision 3.1 software, Thornwood, NY). Digital images were analyzed using Image-Pro Plus version 5.0 (Media Cybernetics, Bethesda, MD). The percentage of immunopositive area (immunopositive area/total intimal area $\times 100$ ) was calculated.

In situ labeling of DNA fragmentation was performed using terminal deoxyribonucleotide transferase (TdT)-mediated nick-end labeling based on an in situ apoptosis detection kit (TACS, Trevigen). Deparaffinized sections were treated with $0.3 \%$ hydrogen peroxide for 10 minutes for inactivation of endogenous peroxidase. The sections were rinsed and then digested with $20 \mu \mathrm{g}$ of proteinase K (EM Science). Exposed DNA fragments were labeled with biotinylated nucleotides (dNTPs) and TDT for 1 hour at $37^{\circ} \mathrm{C}$. The incorporation of biotinylated nucleotides into DNA was detected with a streptavidin-conjugated horseradish peroxidase. A positive reaction was visualized with the chromogenic substance diaminobenzidine tinted with $\mathrm{CoCl}_{2}$, producing a black reaction product. The sections were counterstained with methylgreen (blue-green nuclei). The number of positive cells was counted in the whole intima of each section.

\section{In Vitro Experimental Design}

Cell culture studies. The in vitro experiments were performed with human monocytic leukemic cell line (THP-1). Cells were grown in RPMI medium 1640 supplemented with $10 \%$ heat-inactivated fetal bovine serum, 100 units $/ \mathrm{mL}$ penicillin, $100 \mu \mathrm{g} / \mathrm{mL}$ streptomycin, and $2 \mathrm{mM}$ L-glutamine at $37^{\circ} \mathrm{C}$ in $5 \% \mathrm{CO}_{2}$.

Recombinant plasmid vectors. A full-length cDNA of CASP1 was isolated from ICE- $\alpha$ pBluescript KS plasmid $^{15}$ and was cloned into the Xba1-EcoR1 site of eukaryotic expression vector pCDNA3 (Invitrogen corporation, Carlsbad, CA). Authenticity of the clone was confirmed by DNA sequencing. Empty control vector pcDNA3 and cDNA of CASP1 cloned in reverse orientation into the BamH1-EcoR1 site of pcDNA3 were used as negative controls. 
Electroporation of THP-1 cells. Exponentially growing THP- 1 cells $\left(1 \times 10^{7}\right.$ cells $)$ were washed and resuspended in $300 \mu \mathrm{L}$ complete media containing $20 \mathrm{mM}$ Hepes. Cell suspension was mixed with $10 \mu \mathrm{g}$ of pCDNA3-CASP1, pCDNA3-CASP1-as, or pCDNA3 plasmid DNA and transferred to a cuvette (0.4-cm gap; BioRad Laboratories) and electroporated ( $230 \mathrm{~V}, 960 \mu \mathrm{F}$, Gene Pulsar II, BioRad Laboratories, Hercules, CA). Electroporated cells were diluted with $10 \mathrm{~mL}$ of RPMI 1640 containing 10\% fetal bovine serum, $100 \mathrm{IU} / \mathrm{mL}$ of penicillin, $100 \mu \mathrm{g} / \mathrm{mL}$ of streptomycin, and $0.6 \mathrm{mg} / \mathrm{mL}$ of glutamine, and grown at $37^{\circ} \mathrm{C}$ for 48 hours. MMP-2 and -9 productions were estimated in the culture medium by gel zymography. ${ }^{16}$ Release of MMP-9 and IL1- $\beta$ in the culture medium was also measured by activity assay and ELISA. The effect of a caspase-1 inhibitor and an ER stress inducer on MMP production was also evaluated.

Gel zymography. Culture supernatants were mixed with SDS-sample buffer (50 mM Tris- $\mathrm{HCl} \mathrm{pH} \mathrm{6.8,} \mathrm{10 \%} \mathrm{glyc-}$ erol, $1 \%$ SDS, $0.01 \%$ bromophenol blue) in the absence of reducing agent and electrophoresed in a 7.5\% SDS-polyacrylamide gel containing $0.1 \%(\mathrm{w} / \mathrm{v})$ gelatin. The gel was incubated at room temperature for 1 hour in $2.5 \%$ TritonX 100 and subsequently at $37^{\circ} \mathrm{C}$ overnight in a buffer containing $[10 \mathrm{mM}$ $\mathrm{CaCl}_{2}, 150 \mathrm{mM} \mathrm{NaCl}$, and $50 \mathrm{mM}$ Tris ( $\left.\left.\mathrm{pH} 7.5\right) 0.02 \% \mathrm{NaN}_{3}\right]$. The gel was then stained for protein with $0.25 \%$ Coomassie. Gelatinolytic activity was detected as a clear zone in a dark field.

Matrix metalloproteinase activity assays. For MMP assay, MMP-9 Biotrak Activity Assay System and Interleukin-1 $\beta$ Biotrak ELISA system (GE Healthcare Bio-Sciences, Piscataway, NJ) were used according to manufacturer's protocol. THP-1 culture medium was collected for MMP assay after 48 hours of transfection or 24-48 hours treatment with different inducers.

Reagents. Cell-permeable caspase-1 inhibitor YVAD$\mathrm{CHO}$, caspase-3 inhibitor DEVD-CHO, and broad-spectrum caspase inhibitor Z-VAD-FMK, (BIOMOL International, Plymouth Meeting, PA) were used. 7-ketocholesterol, cholesterol-5 $\beta$, ionomycin, and thapsigargin were purchased from Sigma Chemical Co. (St. Louis, MO).

\section{Statistical Analyses}

All results are presented as the mean \pm SD. To determine the statistical significance of differences between groups oneway ANOVA was used followed by post hoc analysis using Fisher's PLSD. $P$ value of $<.05$ was considered statistically significant. Blood radioactivity of MPI and AA5 was presented as the mean $( \pm \mathrm{SD})$ percent $\mathrm{ID} / \mathrm{g}$ of tissue and was plotted as a function of time after injection. The clearance curves were fitted to biexponential functions by nonlinear least squares.

\section{RESULTS}

\section{Noninvasive Detection of MMP Expression and Apoptosis in Atherosclerotic Lesions}

In vivo dual radionuclide micro-SPECT/CT study was performed immediately and 4 hours after simultaneous intravenous administration of Tc-MPI and In-AA5.
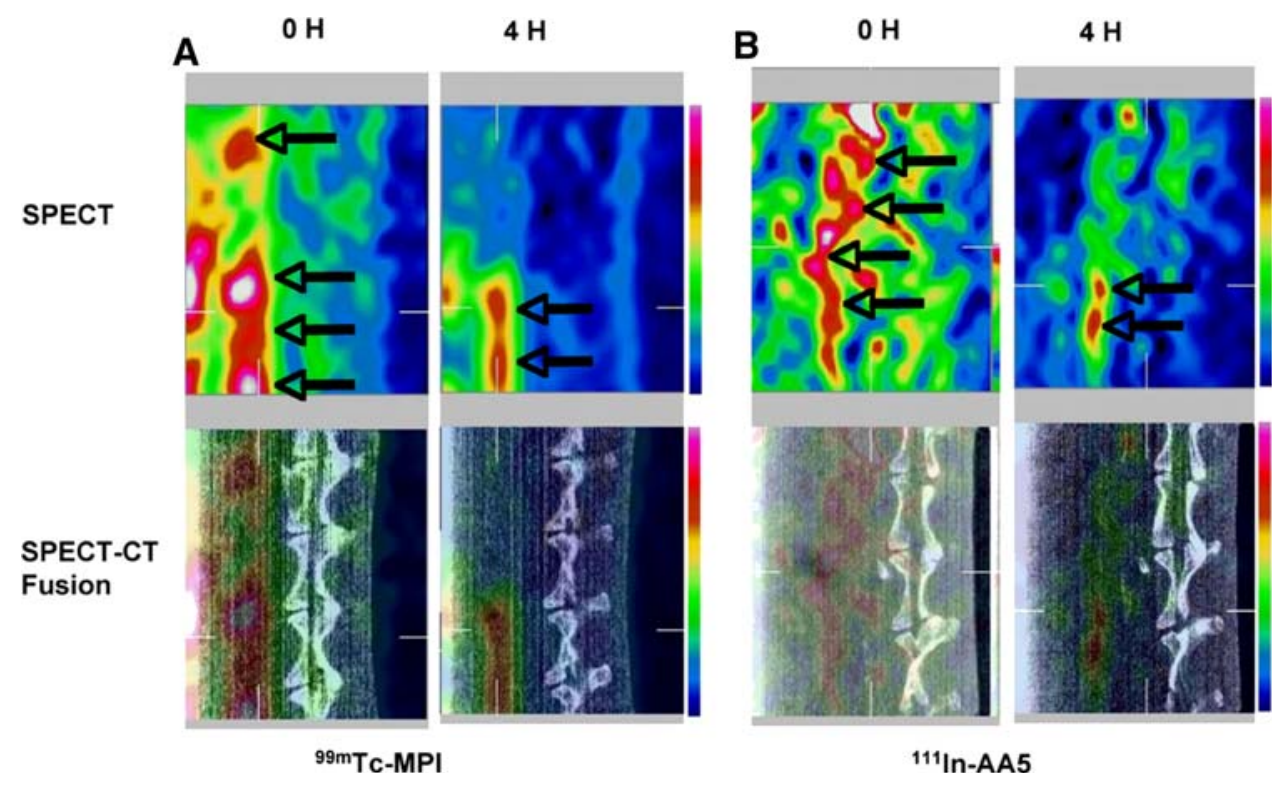

Figure 1. Noninvasive radionuclide imaging of Tc-MPI and In-AA5 in atherosclerosis. MicroSPECT (top), and micro-SPECT-CT fusion images (bottom) of atherosclerotic rabbit on uninterrupted HC diet using Tc-MPI (A) and In-AA5 (B). The left sets in A and B display images immediately ( 0 hour) after radiotracer administration, which reveal blood pool activity in the aorta (arrows) in front of vertebral column. The right sets in $\mathbf{A}$ and $\mathbf{B}$ show target localization $4 \mathrm{~h}$ after radiotracer administration. The radiotracer uptake in the atherosclerotic lesions of the abdominal aorta is observed (arrows). 
The SPECT images obtained at the time of radiotracer injection demonstrated aortic blood pool activity in animals of all study groups. The lesion uptake of both radiotracers was visualized best at 4 hours after tracer administration in the abdominal aorta of animals on uninterrupted HC diet (Figure 1). MPI and AA5 uptake was substantially reduced after diet withdrawal and treatment with fluvastatin. No uptake of the radiotracers was seen in unmanipulated disease control animals.

The uptake of both radiotracers was confirmed in the ex vivo gamma images of the excised aortas, which confirmed the in vivo uptake distribution (Figure 2A). Contrary to the negative control, significant radiotracer accumulation was seen in the abdominal aorta of the atherosclerotic animals placed on an uninterrupted $\mathrm{HC}$ diet. MPI and AA5 uptake was clearly reduced in the diet-withdrawal and statin groups, and no uptake was seen in unmanipulated disease control animals.

\section{Quantitative MPI and AA5 Uptake}

The percent ID/g uptake of MPI in the atherosclerotic lesions of the animals on uninterrupted highcholesterol diet $(0.087 \pm 0.018 \%)$ was significantly higher than the uptake in the abdominal aorta of disease control animals $(0.014 \pm 0.004 \% ; \quad P<.0001)$. The quantitative MPI uptake in diet-withdrawal group $(0.047 \pm 0.005 \% ; \quad P<.0005)$ and fluvastatin-treated animals $(0.053 \pm 0.013 \% ; P<.01)$ was significantly lower compared to uninterrupted HC diet group $(0.087 \pm 0.018 \%)$ (Figure $2 \mathrm{~B})$. Similarly, the percent ID/g AA5 uptake in the atherosclerotic lesions of animals with uninterrupted $\mathrm{HC}$ diet $(0.03 \pm 0.01 \%)$ was significantly higher than the uptake in disease control rabbits $(0.0007 \pm 0.0002 \% ; P<.0001)$. The quantitative uptake of AA5 in diet-withdrawal group (0.018 \pm $0.004 \% ; P<.05)$ and fluvastatin-treated groups $(0.018 \pm$ $0.01 \% ; P<.05)$ was significantly lower compared to uninterrupted HC diet group (Figure 2B).

There was a strong correlation between MPI and AA5 uptake in the atherosclerotic lesions $(\mathrm{r}=0.62$, $P<$.0001) (Figure 2C).

\section{Histopathologic Characterization of Atherosclerotic Lesions}

The atherosclerotic lesions in the HC diet group were extensive and predominantly comprised AHA type IV lesions, showing high macrophage content, significant macrophage apoptosis, low SMC prevalence, and upregulation of MMP-9 (Figure 3A). The macrophage content, the incidence of apoptosis, and the MMP expression were markedly reduced in the intervention groups. There was a significant relationship between

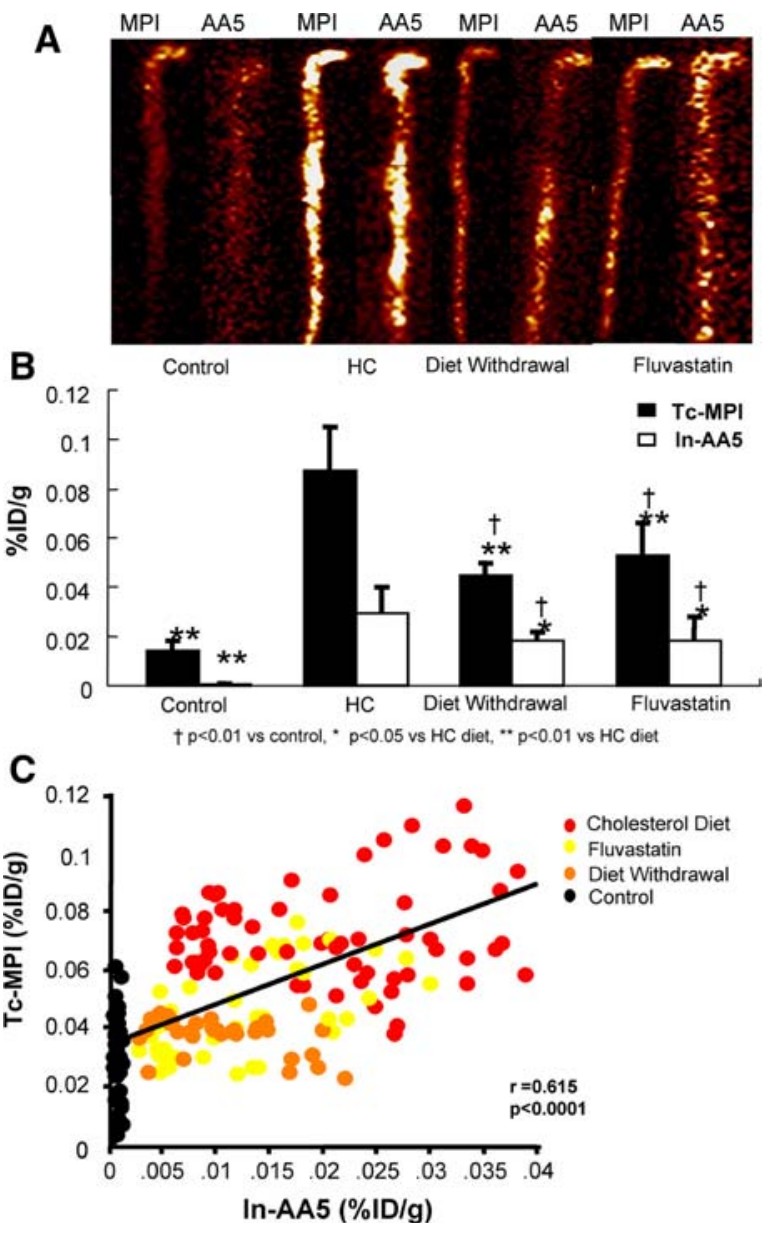

Figure 2. Radiotracer uptake in the aorta by ex vivo imaging and quantitative estimates of Tc-MPI and In-AA5\%ID/g uptake. A Ex vivo images of explanted aortas of the different study groups (control; HC diet; diet withdrawal; fluvastatin) are displayed. Each column shows two images of the same aorta; In-AA5 (right) and Tc-MPI (left). Intense uptake of TcMPI and In-AA5 within the abdominal aortas of rabbits on uninterrupted HC diet is observed. The uptake of both radiotracers is significantly reduced in fluvastatin-treated and diet-withdrawal rabbits. No uptake of both radiotracers is observed in the control animals. B Quantitative \%ID/g Tc-MPI uptake (black bar) and In-AA5 uptake (open bar) of the different study groups is shown. Uptake of both radiotracers is significantly reduced in statin-treated and diet-withdrawal animals compared to the animals on uninterrupted HC diet. C A significant correlation of Tc-MPI and In-AA5 uptake in different groups is observed.

$\%$ ID/g MPI or AA5 uptake and expression of MMP-9 ( $\mathrm{r}=0.786, P<.0001 ; \mathrm{r}=0.562 P<.05$, respectively). The quantitative uptake of both MPI and AA5 was directly related to macrophage content $(\mathrm{r}=0.781$, $P<.0001 ; \quad \mathrm{r}=0.604, \quad P<.01, \quad$ respectively) and TUNEL staining $(\mathrm{r}=0.697, \quad P<.001 ; \quad \mathrm{r}=0.652$, $P<.005$, respectively) (Figure $3 \mathrm{~B}$ ). 
A

CONTROL
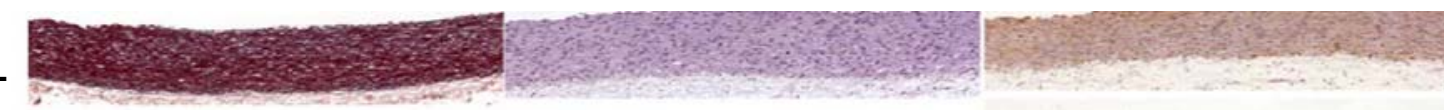

HC DIET

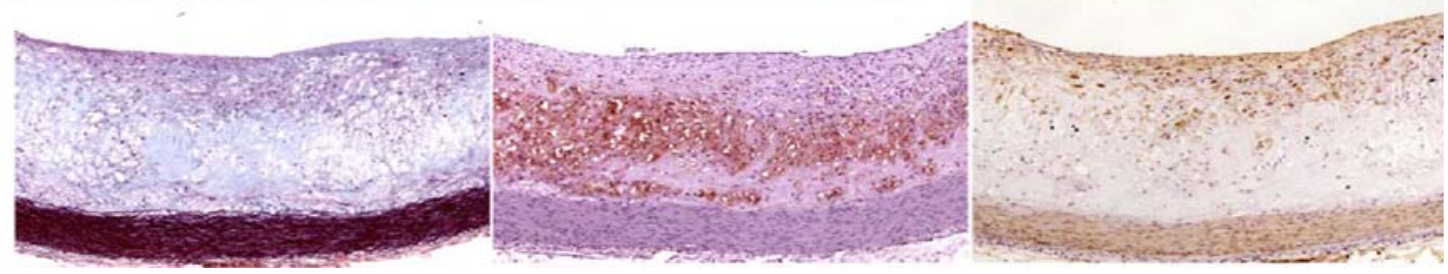

B

RAM-11

MMP-9

TUNEL

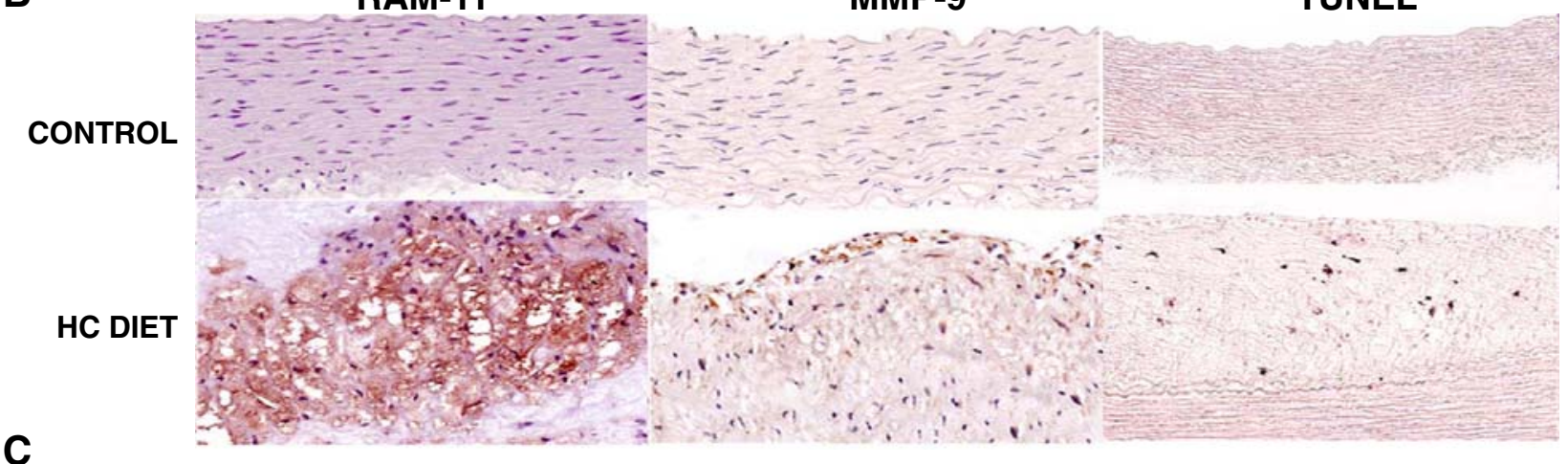

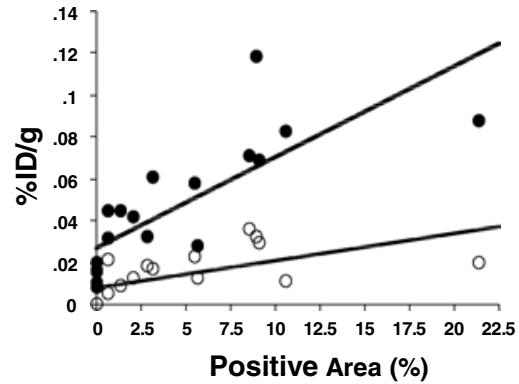

- Tc-MPI

○ In-AA5

$r=0.781$ $p<0.0001$

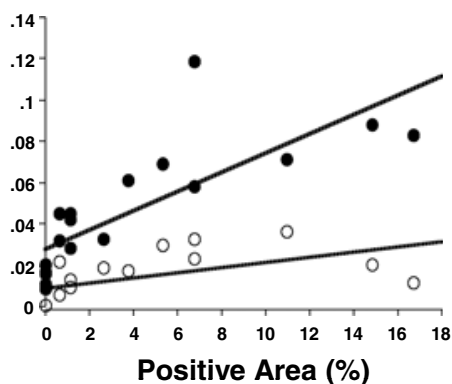

- Tc-MPI

$r=0.786$ $p<0.0001$
- In-AA5

$r=0.562$

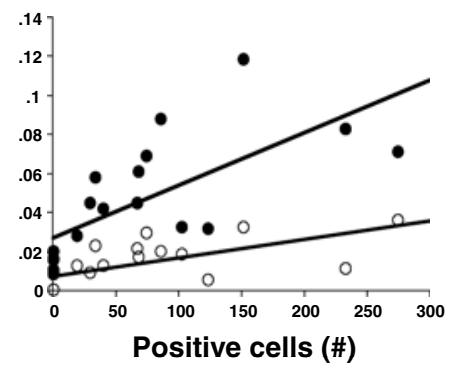

- Tc-MPI

- In-AA5

$r=0.697$ $p<0.001$

Figure 3. Histopathological and immunohistopathological characterization of atherosclerotic lesions in HC diet group compared to control animals. Representative microphotographs from HC diet (bottom) and control animals (top) are shown. A Movat pentachrome, RAM-11 (macrophages), and $\alpha$-actin (smooth muscle cells), B RAM-11 terminal deoxyribonucleotide transferase-mediated nick-end labeling (TUNEL) staining, matrix metalloproteinase MMP-9 immunostaining are shown. Atherosclerotic lesion in the HC group predominantly belongs to AHA type IV lesions and show high macrophage, low SMC, high MMP-9 content. The control group has no atherosclerotic lesions, no apoptosis, and no MMP-9 expression. C Correlation between quantitative Tc-MPI and In-AA5 uptake in $\% \mathrm{ID} / \mathrm{g}$ and pathologically verified positive area of RAM-11, MMP-9, and TUNEL staining. A significant correlation between the quantitative radiotracers uptake and RAM-11, MMP-9, and TUNEL staining are observed.

\section{In Vitro Culture Studies}

Caspase-1 expression in THP-1 cells induced a marked increase in MMP-9 production and extracellular release into the culture medium as assessed by gel zymography (Figure 4A). Cells transfected with empty vector or CASP1 cloned in reverse orientation had no 
effect on MMP-9 expression level. We found a 25-fold increase in MMP-9 activity in CASP1-transfected cells in comparison to the negative controls in bioactivity assay (Figure 4C). There was also a parallel increase in IL1- $\beta$ level in this group (Figure 4D). CASP1 overexpression, however, exerted no effect on MMP-2 level, which remained unchanged in all groups (Figure 4A and B). CASP1-mediated increase in MMP-9 was inhibited by cell-permeable broad-spectrum caspase inhibitor, Z-VAD-FMK, and a CASP1 specific inhibitor, YVAD-CHO (Figure 5A). YVAD-CHO at $1 \mu \mathrm{M}$ concentration reduced the level of MMP-9 release and its activity by more than $50 \%$ (Figure $5 \mathrm{~B}$ and $\mathrm{C}$ ). On the other hand, a caspase-3 inhibitor, DEVD-CHO, had no
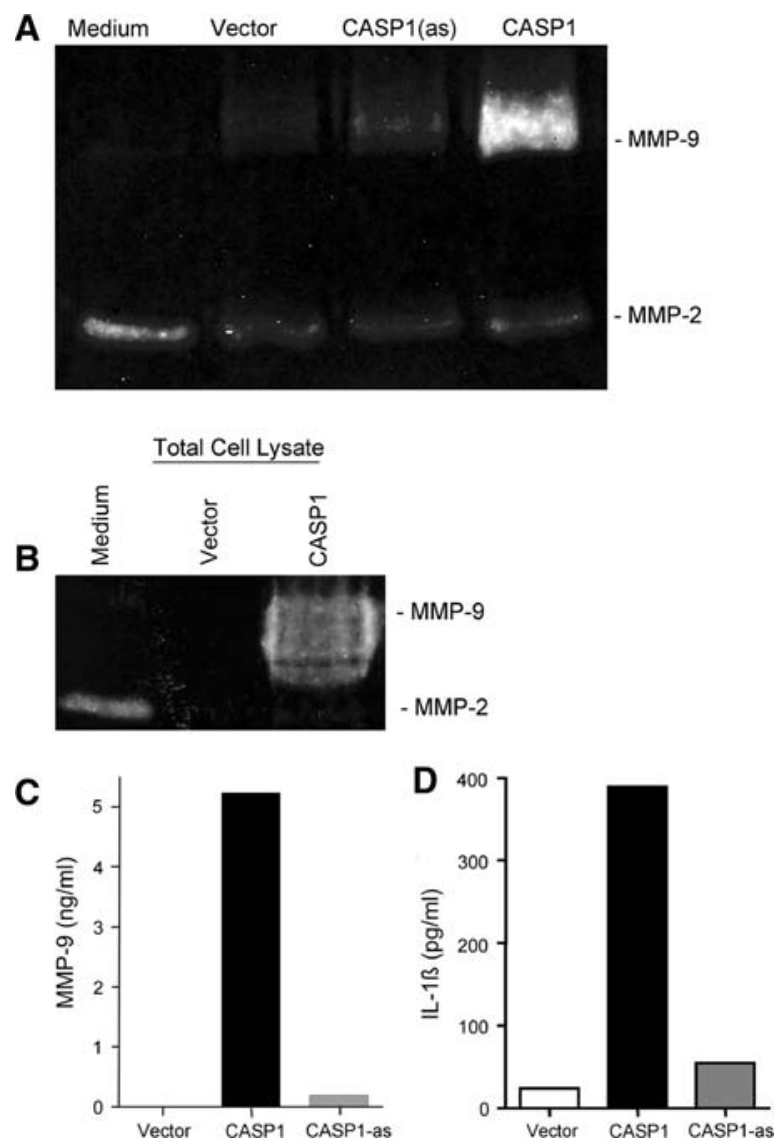

Figure 4. Expression of MMP-9 in CASP1 transfected THP-1 Cells. A Zymogram: caspase-1 transduction of human monocyte THP-1 cells strongly induced MMP-9 expression as analyzed in the culture medium. Cells transfected with empty vector or vector containing CASP1 cloned in reverse orientation [CASP1(as)] did not induce MMP-9 expression. CASP1 expression did not result in MMP-2 expression. B Total cell lysate also reveals MMP-9 in CASP1 transfected THP-1 cells. C Substantially increased level of MMP-9 enzymatic activity is seen in CASP1 expressing THP-1 cells, as is the increased level of IL1- $\beta$ in CASP1 expressing THP-1 cells (D). significant effect on MMP-9 level (Figure 5A), which indicated that CASP1 was primarily responsible for the increase in MMP-9.

We also evaluated the role of oxysterol, cholesterol$5 \beta$, and 7-ketocholesterol, present in atherosclerotic lesions and reported to induce SMC death and plaque destabilization, on MMP-9. THP-1 cells treated with $40 \mu \mathrm{g} / \mathrm{mL}$ of 7 -ketocholesterol and $30 \mu \mathrm{M}$ of cholesterol-5 $\beta$ induced the expression and release of MMP-9 (Figure 5D). Apoptosis induced by oxLDL is mediated through $\mathrm{Ca}^{2+}$ influx and $\mathrm{Ca}^{2+}$ deregulation. THP-1 cells treated with $1 \mu \mathrm{M}$ thapsigargin, an inhibitor of sarcoplasmic reticulum $\mathrm{Ca}^{2+}$ ATPase (SERCA) which raises cytosolic calcium concentration also induced higher MMP-9 release (Figure 5D). But ionomycin, a potent and selective $\mathrm{Ca}^{2+}$ ionophore, which acts primarily at the level of the internal $\mathrm{Ca}^{2+}$ stores and enhances $\mathrm{Ca}^{2+}$ influx, had no effect on MMP-9 expression level (Figure 5D). Lipopolysaccharide (LPS) treatment induced higher MMP-9 expression and extracellular release into the medium, but exactly similar to CASP1 overexpression had no effect on MMP-2 level (Figure 5E). MMP-2 and -9 levels remained unchanged in interferon-gamma (IFN- $\gamma$ )-treated THP-1 cells.

\section{DISCUSSION}

The direct relationship between macrophage apoptosis and MMP release was observed in imaging experiments, which was maintained over the broad range of prevalence of apoptosis and MMP production. Both, the MMP production and apoptosis were substantially reduced in diet-withdrawal and statin treatment groups. MPI and AA5 uptake correlated with pathologically verified apoptosis and macrophage, and MMP-9 positive areas in the atherosclerotic lesions. This relationship was confirmed in culture experiments. Both, in vitro and in vivo experiments demonstrated that macrophage apoptosis and MMP production may be intimately related and highlight the importance of inflammation in plaque vulnerability.

\section{Macrophage Apoptosis and MMP Release}

Although not evaluated in atherosclerosis, neurons under cellular stress undergo apoptosis and release MMP-3. ${ }^{17}$ The MMP-3 upregulation occurs upstream of caspase-3 (CASP3) activation but downstream of c-Jun $\mathrm{N}$-terminal protein kinase (JNK) in the apoptosis signaling cascade. C-Jun, phosphorylated by JNK, acts as a transcription factor for MMP-3 gene. However, our experiments suggest that apoptosis may be upstream to MMP-9 release. Differentiated versus undifferentiated 

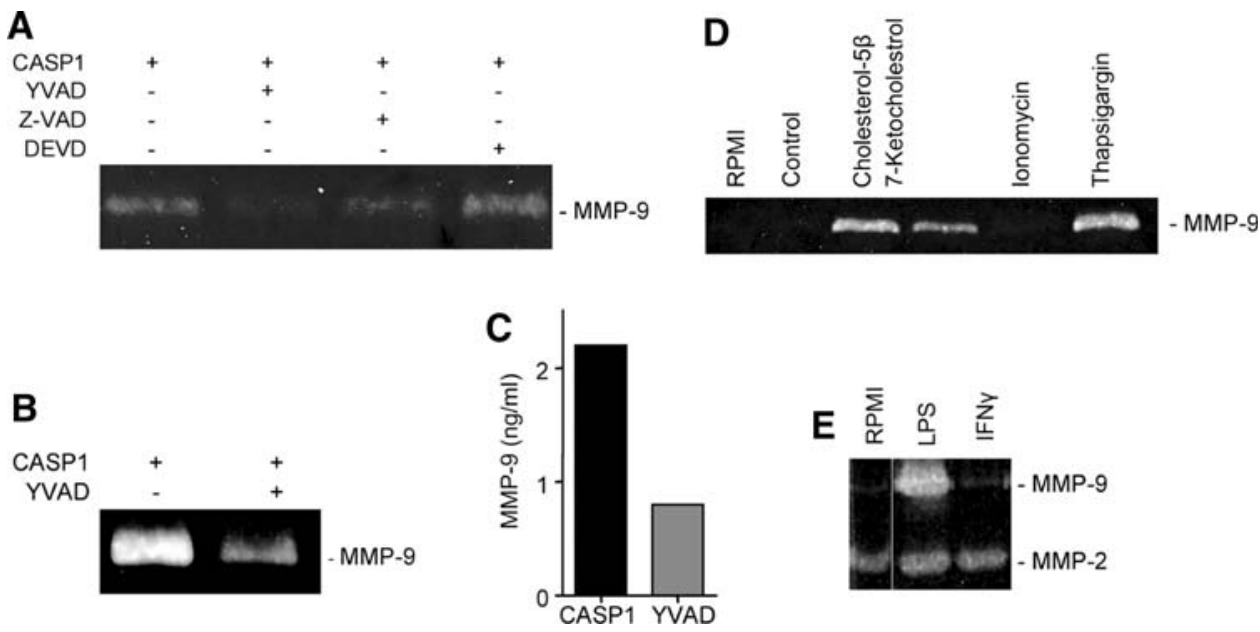

Figure 5. Effect of caspase inhibitors on the expression of MMP-9 in CASP1-transfected THP-1 cells. A Zymogram: Z-VAD-FMK, a cell-permeable broad-spectrum caspase inhibitor and YVADCHO, a CASP1 specific inhibitor, show marked inhibition of MMP-9 release from THP-1 cells. DEVD-CHO, a caspase-3 specific inhibitor, had no effect on MMP-9 expression and release. B, C. MMP-9 enzymatic activity in CASP1 expressing THP-1 cell lysate is significantly reduced by a CASP1 specific inhibitor YVAD-CHO. D Effect of ER stress on the expression of MMP-9 in THP1 cells, as shown by oxysterols, 7-ketocholesterol, and cholesterol-5 $\beta$ induce MMP-9 release from THP-1 cells. SERCA inhibitor thapsigargin also induces MMP-9 expression while it remains unchanged in ionophore ionomycin-treated cells. E Cells treated with LPS release MMP-9 into the medium. IFN $\gamma$ has no effect on MMP-9 expression. MMP-2 level remains unchanged in both groups.

cell types, CASP3 versus CASP1, and MMP-3 versus MMP-9 may explain some of the differences. Our study suggests that CASP1, besides its pro-inflammatory actions, has pro-apoptotic potential. ${ }^{3}$ In patients dying of acute coronary events, extensive apoptosis of macrophages, CASP1 activation, and increased MMP-9 activity have been demonstrated at the site of plaque rupture. $^{2}$ We have also observed CASP1-mediated apoptosis of macrophages in advanced experimental atherosclerosis in a rabbit model. ${ }^{3}$ On the other hand, a possible role of MMP in inducing apoptosis in atherosclerosis is unclear. Besides extracellular matrix proteins, MMP are known to degrade integrins, ${ }^{18}$ and may promote apoptosis of SMC by cell-surface N-cadherin shedding. Also by liberating nonmatrix substrates such as growth factors from attachment to matrix components or cell surface, MMP can influence apoptosis. ${ }^{19}$ MMP can modulate apoptotic signaling by release of FasL ${ }^{20}$ and TNF- $\alpha^{21}$ from cell surface.

Active MMP-3 has also been found in the nucleus of several cultured cell types and may be associated with an increased rate of apoptosis. ${ }^{22}$ Pro-MMP-2 has been found in the nucleus of cardiomyocytes; activated nuclear MMP-2 results in DNA repair enzyme polyADP ribose-polymerase (PARP) fragmentation and may increase genetic instability to trigger apoptosis. ${ }^{23}$ In adrenergic receptor-stimulated rat cardiac myocytes, MMP-2 impairs $\beta 1$ integrin-mediated survival signals, such as activation of focal adhesion kinase (FAK), and activates the JNK-dependent mitochondrial death pathway leading to apoptosis. ${ }^{24}$

\section{Role of Imaging MMP and Apoptosis}

Radiolabeled annexin A5 has been successfully employed for the noninvasive detection of macrophage apoptosis in atherosclerotic plaque ${ }^{10,25}$ and treatmentrelated changes in apoptosis. ${ }^{3}$ Similarly, imaging of MMP activity and treatment-related changes in MMP content within the plaque with a radiolabeled MPI has also been successfully demonstrated previously. ${ }^{9}$ Lipid lowering by dietary modification or HMG-CoA reductase inhibitors, which are associated with an abrogation of apoptosis and a reduction of MMP in plaque are known to stabilize atherosclerotic plaques.

Although not undertaken, it is frequently proposed that multiple tracers may be combined for improving diagnostic accuracy of an imaging procedure for identification of a disease process. Such suggestion is often based on the observed incremental value of combining circulating biomarkers for risk prediction. The direct relationship and similar response to therapeutic 


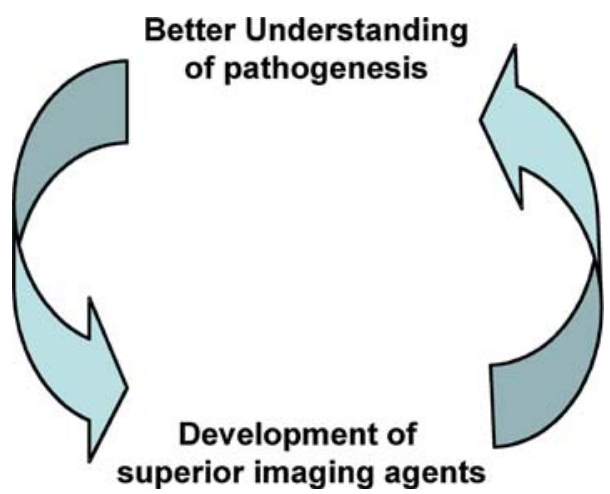

Figure 6. From better understood pathogenesis to development of superior diagnostic strategies, and back. Molecular imaging unravels subcellular mechanisms and does so more effectively since the targeting is undertaken in a living organism.

manipulation in MPI and AA5 uptake suggests that it may not be as advisable to combine two interrelated components of the same pathologic cascade. The incremental value of the diagnostic procedure may be based on combination of morphological and functional imaging or the molecular targeting of the components of different cascades. The example of the latter approach may include functional imaging for identification of an increase in vasa vasorum or neovascularization with either apoptosis or MMP imaging. Nonetheless, the present study suggests that molecular imaging allows better understanding of a pathogenetic process of disease manifestation. On the other hand, knowledge of a pathological mechanism may allow for better development of novel imaging techniques. This uncovers the strength of molecular imaging and may lead to better management and preventive strategies (Figure 6). ${ }^{26}$

\section{CONCLUSIONS}

The present study demonstrates the feasibility of molecular imaging of more than a single target by using different tracers. If the candidate targets are interrelated, dual imaging may not offer sufficient incremental value. Nonetheless, molecular imaging helps understand the pathogenesis of a disease better and vice versa; better understanding of a disease process may allow development of improved diagnostic strategies.

\section{Acknowledgments}

The study was supported by National Institutes of Health grant RO1 (HL 078681) provided to Jagat Narula, MD. He received AA5 from PharmaTarget Inc., Maastricht, Netherlands, MPI from Lantheus Imaging Inc., N. Billerica, $M A$, and fluvastatin from Novartis Pharma and Tanabe Company, Japan.

\section{Open Access}

This article is distributed under the terms of the Creative Commons Attribution Noncommercial License which permits any noncommercial use, distribution, and reproduction in any medium, provided the original author(s) and source are credited.

\section{References}

1. Davies MJ, Thomas A. Thrombosis and acute coronary-artery lesions in sudden cardiac ischemic death. N Engl J Med 1984;310: 1137-40.

2. Kolodgie FD, Narula J, Burke AP, Haider N, Farb A, Hui-Liang $\mathrm{Y}$, et al. Localization of apoptotic macrophages at the site of plaque rupture in sudden coronary death. Am J Pathol 2000;157: 1259-68.

3. Sarai M, Hartung D, Petrov A, Zhou Y, Narula N, Hofstra L, et al. Broad and specific caspase inhibitor-induced acute repression of apoptosis in atherosclerotic lesions evaluated by radiolabeled annexin A5 imaging. J Am Coll Cardiol 2007;50:2305-12.

4. Shah PK, Falk E, Badimon JJ, Fernandez-Ortiz A, Mailhac A, Villareal-Levy G, et al. Human monocyte-derived macrophages induce collagen breakdown in fibrous caps of atherosclerotic plaques. Potential role of matrix-degrading metalloproteinases and implications for plaque rupture. Circulation 1995;92:1565-9.

5. Bjorkerund S, Bjorkerund B. Apoptosis is abundant in human atherosclerotic lesions, especially in inflammatory cells (macrophages and $\mathrm{T}$ cells), and may contribute to the accumulation of gruel and plaque instability. Am J Pathol 1996;149:367-80.

6. Kolodgie FD, Burke AP, Farb A, Gold HK, Yuan J, Narula J, et al. The thin-cap fibroatheroma: A type of vulnerable plaque: The major precursor lesion to acute coronary syndromes. Curr Opin Cardiol 2001;16:285-92.

7. Galis ZS, Khatri JJ. Matrix metalloproteinases in vascular remodeling and atherogenesis: The good, the bad, and the ugly. Circ Res 2002;90:251-62.

8. Schaub FJ, Han DKM, Liles WC, Adams LD, Coats SA, Ramachandran RK, et al. Fas/FADD-mediated activation of a specific program of inflammatory gene expression in vascular smooth muscle cells. Nat Med 2000;6:790-6.

9. Fujimoto S, Hartung D, Ohshima S, Edwards DS, Zhou J, Yalamanchili $\mathrm{P}$, et al. Molecular imaging of matrix metalloproteinase in atherosclerotic lesions: Resolution with dietary modification and statin therapy. J Am Coll Cardiol 2008;52:1847-57.

10. Kolodgie FD, Petrov A, Virmani R, Narula N, Verjans JW, Weber DK, et al. Targeting of apoptotic macrophages and experimental atheroma with radiolabeled annexin V: A technique with potential for noninvasive imaging of vulnerable plaque. Circulation 2003; 108:3134-9.

11. Su H, Spinale FG, Dobrucki LW, Song J, Hua J, Sweterlitsch S, et al. Noninvasive targeted imaging of matrix metalloproteinase activation in a murine model of postinfarction remodeling. Circulation 2005;112:3157-67.

12. Xue CB, Voss ME, Nelson DJ, Duan JJ, Cherney RJ, Jacobson IC, et al. Design, synthesis, and structure-activity relationships of macrocyclic hydroxamic acids that inhibit tumor necrosis factor alpha release in vitro and in vivo. J Med Chem 2001;44:2636-60.

13. Stary HC, Chandler AB, Glagov S, et al. A definition of initial, fatty streak, and intermediate lesions of atherosclerosis. A report from the Committee on Vascular Lesions of the Council on Arteriosclerosis, American Heart Association. Arterioscler Thromb 1994;14:840-56. 
14. Isobe $\mathrm{S}$, Tsimikas $\mathrm{S}$, Zhou J, Fujimoto S, Sarai M, Branks MJ, et al. Noninvasive imaging of atherosclerotic lesions in apolipoprotein E-deficient and low-density-lipoprotein receptor-deficient mice with annexin A5. J Nucl Med 2006;47:1497-505.

15. Alnemri ES, Fernandes-Alnemri T, Litwack G. Cloning and expression of four novel isoforms of human interleukin-1 beta converting enzyme with different apoptotic activities. J Biol Chem 1995;270:4312-7.

16. Lelongt B, Trugnan G, Murphy G, Ronco PM. Matrix metalloproteinases MMP2 and MMP9 are produced in early stages of kidney morphogenesis but only MMP9 is required for renal organogenesis in vitro. J Cell Biol 1997;136:1363-73.

17. Choi DH, Kim EM, Son HJ, Joh TH, Kim YS, Kim D, et al. A novel intracellular role of matrix metalloproteinase-3 during apoptosis of dopaminergic cells. J Neurochem 2008;106:405-15.

18. Frisch SM, Screaton RA. Anoikis mechanisms. Curr Opin Cell Biol 2001;13:555-62.

19. Newby AC. Matrix metalloproteinases regulate migration, proliferation, and death of vascular smooth muscle cells by degrading matrix and non-matrix substrates. Cardiovasc Res 2006;69:614-24.

20. Kayagaki N, Kawasaki A, Ebata T, Ohmoto H, Ikeda S, Inoue S, et al. Metalloproteinase-mediated release of human Fas ligand. J Exp Med 1995;182:1777-83.
21. Gearing AJ, Beckett P, Christodoulou M, Churchill M, Clements J, Davidson AH, et al. Processing of tumour necrosis factor-alpha precursor by metalloproteinases. Nature 1994;370:555-7.

22. Si-Tayeb K, Monvoisin A, Mazzocco C, Lepreux S, Decossas M, Cubel G, et al. Matrix metalloproteinase 3 is present in the cell nucleus and is involved in apoptosis. Am J Pathol 2006;169:1390-401.

23. Kwan JA, Schulze CJ, Wang W, Leon H, Sariahmetoglu M, Sung M, et al. Matrix metalloproteinase-2 (MMP-2) is present in the nucleus of cardiac myocytes and is capable of cleaving poly (ADP-ribose) polymerase (PARP) in vitro. FASEB J 2004; 18:690-2.

24. Menon B, Singh M, Ross RS, Johnson JN, Singh K. beta-Adrenergic receptor-stimulated apoptosis in adult cardiac myocytes involves MMP-2-mediated disruption of beta1 integrin signaling and mitochondrial pathway. Am J Physiol Cell Physiol 2006;290: C254-61.

25. Kietselaer BL, Reutelingsperger CP, Heidendal GA, Daemen MJ, Mess WH, Hofstra L, et al. Noninvasive detection of plaque instability with use of radiolabeled annexin A5 in patients with carotid-artery atherosclerosis. N Engl J Med 2004;350:1472-3.

26. Narula J, Dilsizian V. From better understood pathogenesis to superior molecular imaging, and back. J Am Coll Cardiol Imaging 2008;1:406-409. 